\title{
LIPOPROTEíNAS DE BAJA DENSIDAD (LDL) EN ESTUDIOS EXPERIMENTALES EN CONEJOS hIPERCOLESTEROLÉMICOS
}

Lidia Cruz Neyra

\begin{abstract}
RESUMEN
Las lipoproteínas son macromoléculas metabólicamente diferentes, con una determinada densidad, composición química, características de flotación y movilidad electroforética. Particularmente las Lipoproteínas de Baja Densidad se les atribuye un efecto aterogénico, siendo importante la utilización de animales como modelo experimental para su estudio. El propósito de esta investigación es revisar de manera preliminar los trabajos que incluyen como modelo experimental al conejo, usado en la inducción experimental de hipercolesterolemia $\mathrm{y}$ aterosclerosis.
\end{abstract}

Palabras clave: inducción de hipercolesterolemia, lipoproteinas, conejos.

\section{SUMMARY}

Lipoproteins are metabolically different macromolecules with, chemical composition, density flotation and electrophoretic mobility characteristics. Particularly Low Density Lipoproteins (LDL) are attributed atherogenic effect, being important the use of animals as experimental models for study. The purpose of this research is a preliminary review of the work including the rabbit as an experimental model, used in experimentally induced hypercholesterolemia and atherosclerosis.

Keywords: hypercholesterolemia induction, lipoproteins, rabbit.

\section{LíPIDOS Y LIPOPROTEíNAS}

Los lípidos como son los fosfolípidos, triglicéridos, colesterol, entre otros son insolubles en agua y siendo el plasma altamente polar tienen que asociarse a proteínas específicas, llamadas apoproteínas, formando las lipoproteínas y de esta manera pueden circular por la sangre. La clasificación de las lipoproteínas se realiza con respecto a la proporción de lípidos y proteínas, lo que determina su densidad y comportamiento en circulación; cuanta más concentración de lípidos tenga una lipoproteína, menor será su densidad.

Las lipoproteínas son macromoléculas metabólicamente diferentes, con una determinada densidad, composición química, características de flotación y movilidad electroforética. Así tenemos a las Lipo- proteínas de Alta Densidad o High Density Lipoprotein (HDL), Lipoproteínas de Baja Densidad o Low Density Lipoprotein (LDL), Lipoproteínas de Densidad Intermedia (IDL) Lipoproteínas de Muy Baja Densidad o Very Low density Lipoprotein (VLDL), Quilomicrones (QM). (Tsimikas \& Mooser, 2002)

Quilomicrones. Son partículas con densidad de flotación $<0,95 \mathrm{mg} / \mathrm{dL}$, se forman a partir de la degradación y absorción de las grasas de la dieta en el intestino, las que transportan de 85 a $95 \%$ de triglicéridos exógenos, son relativamente pobres en colesterol $(5 \%)$ y fosfolípidos (7\%), y contienen un $2 \%$ de proteínas fundamentalmente apo B-48. Estos llevan tan escasa cantidad de colesterol que su aumento (dislipidemia tipo I) no se asocia a lesiones de ateroma. 
Lipoproteínas de Alta Densidad (HDL) son las lipoproteínas de mayor densidad, las cuales se encargan de recoger los excedentes de fosfolípidos y ésteres de colesterol y llevarlos de nuevo al hígado. Tienen una proporción de 50\% de lípidos y $50 \%$ de proteínas, se forman en el hígado e intestino delgado. Las HDL formadas en el hígado pueden ser HDL-2 que tienen una densidad de 1,063-1,125 $\mathrm{mg} / \mathrm{dL}$ y un contenido de colesterol de $40 \%$ con un $6 \%$ de triglicéridos; mientras que HDL-3 tienen una densidad de 1,125-1,210 mg/dL, su tamaño es menor que la HDL-2, con 33\% de fosfolípidos, 7 $\%$ de triglicéridos y $38 \%$ de colesterol. A las HDL se les atribuye un efecto antiaterogénico, dado el transporte reverso de colesterol de los tejidos periféricos al hígado para su posterior eliminación biliar (Glomset, 1968).

Lipoproteínas de Baja densidad (LDL) se encargan de transportar esteres de colesterol a los tejidos extrahepáticos. Se forman a partir de las VLDL una vez éstas han perdido lípidos por lo que su densidad será ligeramente mayor ytendran un $80 \%$ de lípidos y un $20 \%$ de proteínas. Constituye el $50 \%$ total de lipoproteínas del plasma con una densidad promedio de $1,019-1,063 \mathrm{mg} / \mathrm{dL}$ y compuestas en su mayoría por $50 \%$ de colesterol, $6 \%$ de triglicéridos y $22 \%$ de fosfolípidos y proteínas (apo B-100). El hígado tiene receptores de alta afinidad que reconocen la apo B-100 como la única apolipoproteína presente en la LDL; $50 \%$ de su catabolismo se hace por depuración hepática, función antagónica a HDL que se sintetizan en el intestino y el hígado; por ello, las LDL son las principales portadoras de colesterol en lesiones ateromatosas, al igual que las VLDL.

Lipoproteínas de Muy Baja densidad (VLDL) son partículas más pequeñas que los quilomicrones, producidas por el hígado y tienen una densidad promedio de $0,96-1,006 \mathrm{mg} / \mathrm{dL}$; presentan $60 \%$ de triglicéridos, $12 \%$ de colesterol, $20 \%$ de fosfolípidos y $10 \%$ de proteínas, siendo principalmente apo B-100.

\section{LDL OXIDADA}

En la actualidad existe un consenso del rol del estrés oxidativo en la aterosclerosis que representa un mayor estado de oxidación de lípidos y proteínas en la pared vascular. (Witztum, 1994, Stocker et al. 2004)

La hipótesis de la modificación oxidativa en la aterosclerosis predice que la oxidación de las lipoproteínas de baja densidad (LDL) es un evento temprano en la aterosclerosis y su modificación oxidada contribuye a la formación de ateromas. En sustento a esta hipótesis, se evidencia in vitro que LDL oxidada forma células espumosas (foam cell) e in vivo tiene una serie de actividades potencialmente proaterogénica, y varias de ellas estructuralmente no vinculados a la inhibición por antioxidantes en aterosclerosis en animales. Un nuevo consenso en la enfermedad vascular destaca la importancia de los eventos oxidativos, además de la oxidación del LDL (Aviram, 1996).

Los eventos oxidativos incluyen la producción de especies reactivas de oxígeno y nitrógeno por las células vasculares, así como las modificaciones oxidativas que contribuyen a importantes manifestaciones clínicas de las enfermedades cardiovasculares, tales como la disfunción endotelial y la interrupción de la placa. A pesar de estos abundantes datos sin embargo, siguen habiendo problemas fundamentales con la implicancia de la modificación oxidativa como un requisito patofisiologicamente importante de la aterosclerosis (Ylä-Herttuala, 1991).

El daño oxidativo que se produce por el desequilibrio entre fenómenos antioxidantes/proxidantes parece crucial en el origen de la ateroesclerosis, y esto aumenta la posibilidad que los antioxidantes, como la vitamina $\mathrm{C}$, betacaroteno y en especial el alfa tocoferol (vitamina E), puedan prevenir o retardar el desarrollo de esta enfermedad (Miller, 1998, Stocker \& Keaney, 2004)

\section{ATEROSCLEROSIS Y LDL OXIDADA}

La teoría de la respuesta a la injuria o teoría de respuesta a la lesión oxidativa formulada por Ross $(1976,1986)$ considera que una lesión inicial del endotelio sea el hecho crucial para desencadenar el proceso de aterogénesis. Una lesión en el endotelio puede suceder por diversos factores, entre ellos, radicales libres, aumento de LDL, tensión hemodinámica. Cuando estos factores coexisten, actúan sinérgicamente sus efectos se ven multiplicados amplificando la magnitud de la lesión (Raij, 1991).

Esta teoría es la más aceptada, la LDL penetra en la región de la capa íntima arterial donde ocurre una serie de eventos que involucran los macrófagos, a las células musculares lisas que liberan enzimas y otras sustancias como un intento de proteger de la acción de los metabolitos que oxidan a la LDL.

Las evidencias sugieren que la generación de células espumosas es un proceso independiente de los receptores normales para LDL, presentes en todos los tejidos. Se ha observado en pacientes y 
en animales con deficiencia genética de receptores de LDL desarrollar las lesiones aterosclerótica rica en células espumosas. Brown y Goldstein (1983) reportaron que la captación arterial de LDL ocurre por vías alternativas que fueron denominadas "scavenger pathways", postulando que las LDL circulantes sufrirían algún tipo de transformación en la pared vascular y de esa forma LDL modificada sería fagocitada por los macrófagos y formarían las células espumosas.

\section{MODELO EXPERIMENTAL EN CONEJOS}

Numerosas investigaciones han sido realizadas utilizando modelos experimentales para estudiar el desarrollo de la aterosclerosis con dieta induciendo hiperlipidemia. Debido al hecho de que los conejos son muy sensibles a la dieta ricas en colesterol y acumulan grandes cantidades en el plasma, la utilización de estos animales como modelo experimental para evaluar el desarrollo de aterosclerosis es de gran relevancia, contribuyendo a la información de progresión y regresión aplicadas a situaciones humanas.

Steinberg (2004) en su obra sobre la patogénesis de la aterosclerosis nos relata que Nikolai $\mathrm{N}$. Anitschkow en1914, fue uno de los primeros en inducir hipercolesterolemia en conejos usando yema de huevo, detectando depósitos de grasa en las arterias; pero sus experimentos no fueron bien comprendidos, pues el nivel de colesterol alcanzaba $1000 \mathrm{mg} / \mathrm{ml}$, valores totalmente diferentes de humanos.

Vesselinovith et al (1976) y Wissler et al (1985) realizaron estudios experimentales observando que la disminución del contenido de lípidos está relacionada con la regresión de la placa de aterosclerosis. El trabajo de Badimon et al (1990) mostro que en conejos hipercolesterolemicos, a los cuales se les había administrado HDL se producía una reducción significativa de la concentración de colesterol total, esterificado y libre, así como de fosfolípidos en la pared arterial en relación con el grupo de conejos hipercolesterolémicos que no habían sido tratados.

En los estudios de Ribeiro et al (1994) demostró que tratando a conejos hipercolesterolémicos con estatinas como sinvastatina y pravastatina, disminuyeron los valores de colesterol en la pared arterial, el número de células espumosas y la peroxidación lipídica, así como una reversión de la disfunción endotelial.
Por otro lado, los estudios de Helf et al (2002) usando resonancia magnética demostró que existe regresión y progresión de ateromas en conejos tratados con colesterol por seis meses y regresión por otros seis meses, observando modificación de la placa conforme el tiempo sea más largo o corto de la suspensión de la dieta.

El uso de la espectroscopia de rayos infrarrojo para analizar la placa de aterosclerosis reportado por Moreno et al (2002) posibilita nuevas perspectivas para el conocimiento de la formación y regresión del ateroma.

Ribeiro et al (1994) reportaron que la paravastatina produce un relajamiento dependiente del endotelio de mayor magnitud y disminución del número de células espumosas. Otros efectos adicionales de la pravastatina ocurren, denominándose efectos pleitotropicos.

Para inducir hipercolesterolemia en animales, se han utilizado dietas conteniendo colesterol, las cuales varían de raciones comerciales suplementadas con niveles diferentes de colesterol, así como modificaciones en las porciones de lípido y carbohidrato y en las diferentes fuentes y contenidos de grasa, conteniendo o no ácido cólico (Lichtman et al 1999). Generalmente se induce hipercolesterolemia en conejos alimentados con raciones comerciales suplementadas con $1 \%$ de colesterol para investigar los efectos de potenciales de fármacos (Oliveira et al, 2001; Lima et al, 2001, Percegoni et al, 2004)

Ozaki, (2011) ha reportado que el uso de la estatina rosuvastatina en conejos hipercolesterolémicos disminuyeron los valores de colesterol en la pared arterial, el número de células espumosas y la peroxidación lipídica, así como mostró una reversión de la disfunción endotelial.

Dado que la prevalencia de aterosclerosis y enfermedad cardíaca isquémica está aumentando en el mundo, ocasionando serias consecuencias clínicas que exigen esfuerzos para mejorar el conocimiento de su patogénesis, por lo que es necesario estudiar e implementar mejoras en las técnicas experimentales $\mathrm{y}$ en los nuevos tratamientos que exigen el uso de modelos experimentales. Los modelos experimentales usando conejos han evidenciado una importante herramienta para el estudio de la aterosclerosis, no obstante debe continuar la búsqueda de nuevas técnicas. 


\section{REFERENCIA BIBLIOGRÁFICA}

Almeida EA, Ozaki MR, Ribeiro Jorge PA. Effects of fluvastatin on lipid, lipid peroxidation and endothelial dysfunction in hypercholesterolemic rabbits. J Braz

Aviram, M. 1996. Interaction of oxidized low density lipoprotein with macrophages in atherosclerosis, and the antiatherogenicity of antioxidants. Eur. $J$. Clin. Chem. Clin. Biochem, 34(8):599-608.

Badimon JJ, Badimon L, Fuster V. Regression of atheroma lesions by high density lipoprotein plasma fractions in the cholesterol rabbits. J Clin Invest.1990;85(4):1234-41.

Brown MS, Goldstein JL. Lipoproteins metabolism in the macrophage implications for cholesterol deposition in atherosclerosis. Annual Review of Biochemistry.1983;52:223-61.

Ellison RC, Zhang Y, Qureshi MM, Knox S, Anett DK, Province M. Investigators of the NHLBI Family Heart Study. Lifestyle determinants of high-density lipoprotein cholesterol: the National Heart, Lung, and Blood Institute family heart study. Am Heart J. 2004;147:529-35

Glomset JA. The plasma lecithin: cholesterol acyltranferase reaction. J Lipid Res 1968;9:15567

Helf G, Worthley SG, Fuster V, Fayad ZA, Zaman AG, Corti R. Progression and regression of atherosclerotic lesions: monitoring with serial noninvasive magnetic resonance imaging. Circulation. 2002;105:993-8.

Lichtman AH, Clinton SK, Iyama K, Connelly PW, Libby P, Cybulsky MI. Hyperlipidemia and atherosclerotic lesion development in LDL receptordeficient mice fed defined semipurified diets with and without cholate. Arterioscler Thromb Vasc Biol. 1999; 19 (8): 1938-44.

Lima LRP, Oliveira TT, Nagem TJ, Pinto AS, Stringheta PC, Tinoco ALA, et al. Bixina, norbixina e quercetina e seus efeitos no metabolismo lipídico de coelhos. Braz J Vet Res Anim Sci. 2001; 38 (4): 196-200.

Miller III ER, Appel LJ, Risby TH. 1998. Effect of dietary patterns on measures of lipid peroxidation. Circulation, 98:2390-2395.

Moreno PR, Lodder RA, Purusshothaman KR, Charash WE, Oconnor WN, Muller JE. Detection of lipid pool thin fibrous cap and inflamatory cells in human aortic atherosclerotic plaque by near-infrared spectroscopy. Circulation. 2002;105:923-27.

Oliveira TT, Nagem TJ, Pinto AS, Lopes RM, Camini NA, Leão MA, et al. Ação de chitosan e naringenina em coelhos hiperlipidêmicos. Rev Portuguesa de Farmácia. 2001; 1: 43-8.

Ozaki. Evolução e involução da aterosclerose: Estudo experimental em coelhos hipercolesterolêmicos. Tesis de doctorado. 2011. Universidad Estadual de Campinas. S.P.

Percegoni N, Oliveira TT, Nagem TJ, Lima LRP, Lima EQ, Matta SLP. Efeito de caseína, proteína isolada de soja e colestiramina em coelhos hiperlipidêmicos. Rev Cienc Farm. 2004; 25: 99-110.

Raij L.Hypertension, endothelium, and cardiovascular risk factors. Am J Med.1991;90(suppl 2A):13-8.

Ribeiro Jorge P, Ozaki MR, Almeida EA, Metze $\mathrm{K}$, Credidio Neto L. Effects of vitamin E on endothelium-dependent coronary flow in hypercholesterolemic dogs. Atherosclerosis. 1996;126:43-51

Ribeiro Jorge P, Ozaki MR, Almeida EA. Endothelial dysfunction in coronary vessels and thoracic aorta of rats exposed to cigarrete smoke. Clinical and Experimental Pharmacology \& Physiology. 1995;22(Ribeiro Jorge PA, Ozaki MR, Almeida EA. Endothelial dysfunction in coronary vessels and thoracic aorta of rats exposed to cigarrete smoke.. Clinical and Experimental Pharmacology \&Physiology v. 22, p. 410-413, 1995.):410-3.

Ribeiro Jorge P, Ozaki MR, Metze K. Effects of pravastatin and sinvastatin on endothelium dependent relaxation in hyphercholestrolemic rabbits. Exp Tox Pathol 1994;46:465-9.

Ribeiro Jorge PA, Almeida EA, Ozaki MR, Jorge M, Carneiro A. Ribeiro Jorge PA, Almeida EA, Ozaki MR, Jorge M, Carneiro A. Efeitos da Atorvastatina, Fluvastatina, Pravastatina e Simvastatina sobre a funcao endotelial, a peroxidacao lipidica e a ateroscleroseaortica em coelhos hipercolesterolemicos. Arquivos Bras Cardiol 2005;84(4):314-9.

Ribeiro Jorge PA, Neyra LC, Ozaki MR, Almeida EA. Ribeiro Jorge PA, Neyra LC, Ozaki MR, Almeida EA. Improvement in the endothelium-dependent relaxation in hypercholesterolemic rabbits treated with vitamin E. Atherosclerosis.1998;40(2):333-9 
Ross R, Harker L. Hyperlipidemia and atherosclerosis. Science. 976;193:1094-1100.

Ross R. The pathogenesis of atherosclerosis. N Engl J Med. 1986;314:488-500

Society of Int Med. 2004;2(3):63-71.

Steinberg D. Thematic review series: the Pathogenesis of atherosclerosis. An interpretative history of the cholesterol controversy: part I. Journal of Lipid Research. 2004;45:1583-93.

Tsimikas S, Mooser V. Molecular Biology of Lipoproteins and Dislipidemias in Molecular Basic Cardiovascular Disease. 2 nd ed. Philadelphia: W B Saunders Company; 2002.p.1008-11
Vesselinovith D, Wissler RW, Hughes R, Borensztajn J. Reversal of advanced atherosclerosis in Rhesus monkeys Light microscopic studies Atherosclerosis.1976;23:155-76.

Wissler RW, Vesselinovith D, Davis HR, Lambert $\mathrm{PH}$, Bekermeier M. A new way to look at atherosclerotic involvement of the artery wall and the functional effects. Ann N Y Acad Sci. 1985;454:9-22.

Witztum JL. 1994. The oxidation hypothesis of atherosclerosis. Lancet 344:793-795.

Ylä-herttuala, S. (1991) Macrophages and oxidized low density lipoproteins in the pathogenesis of atherosclerosis. Ann. Med., 23(5):561-567. 